\title{
Onkologie 1987;10:108
}

\section{Buchbesprechungen}

Mineralien und Spurenelemente in Klinik und Praxis, Bd. 3 Mineralstoffwechsel beim

Tumorpatienlen

Herausgeber: W. Bayer, K.-H. Schmidt

Verlag für Medizin Dr. Ewald Fischer GmbH, Heidelberg

1984

92 S., 7 Abb., 4 Tab., kartoniert, DM 22-

Es handelt sich um den Band 3 der Schriftenreihe «Mineralien und Spurenelemente in Klinik und Praxis». In ihm sind die 6 Referate wiedergegeben, die während einer Tagung anläßlich der Medizinischen Woche in Baden-Baden 1983 gehalten wur-den. Die auf 77 Seiten behandelten Themen betreffen, abgese-hen von allgemeinen Beobachtungen und Überlegungen über Mineralstoffwechsel und Tumorentstehung bzw. -wachstum, mögliche Beziehungen zwischen Magnesiummangel und Kar-zinomen, die Rolle des Selens bei malignen Erkrankungen, die Wirkungen von Tumoren auf den Kupfer- und Eisenstoff-wechsel und schließlich Steroide und Komplementfaktoren in der Krebsabwehr und deren mögliche Beeinflussung durch Zinkaspartat. Die Vorträge sind überwiegend als Übersichts-referate angelegt, in einigen werden auch kasuistische Mittei-lungen über einzelne Patienten gemacht. Kontrollierte klini-sche Studien zu den aufgeworfenen Fragen fehlen völlig. Aus den Referaten geht hervor, daß viele vermutete Beziehungen zwischen Tumorgeschehen und Spurenelementen noch in das Gebiet der Spekulation fallen. In einigen Punkten, z.B. beim Selen, sind aber auch Ansätze für definierte Fragestellungen und damit für eine gezielte Forschungsarbeit zu erkennen.

O. Heídenreich, Aachen

ist, daß die Marknagelung als solche eine Renaissance erlebi sondern wegen der Unmittelbarkeit der Übertragung vo Änderungen durch die eigene Handschrift. Zum Buch selbs ist wenig zu sagen. Es ist nach wie vor voll gültig und gliedei sich in einen allgemeinen und speziellen Teil und in die Kapite Einführung, Gefahren der Marknagelung, Indikationen de Marknagelung, Technik der Marknagelung, Technik de Marknagelung des Knochenbruchs, Arthrodese mittels Mark nagelung, Arthrorise, Arthroplastik, die Marknagelung de Spontanfraktur, die Nagelung des gelenknahen Bruchs, dii Marknagelung der Pseudarthrose, die Osteotomie, die Kno chentransplantation, die Marknagelung im Tierversuch un < die Nachbehandlung. Die Aussagen dieses Werkes beinhaltei den Erfahrungsschatz eines dafür sehr reichen Lebens un( sind auch heute noch uneingeschränkt gültig, wenngleich bei spielsweise im Kapitel der Knochentransplantation die Aus sage, daß die Knochentransplantation nur ganz seiten in Frag $₫$ komme und daß der Span stets zugrunde gehe, eine Akzent verschiebung erfahren hat. Das Buch ist 349 Seiten stark, die auf Glanzpapier wieder gegebenen Abbildungen sind einwandfrei, und neben derr aktuellen therapeutischen Wert besitzt das Werk eine herausragende medizinhistorische Bedeutung. Es sollte vor alien Dingen bei den Fachleuten, das sind Chirurgen, Unfallchirur-gen und Orthopäden, ebensowenig fehlen wie bei Medizin-historikern, und es gehört in jede Fachbibliothek.

H. Ecke, Gießen 
Praxis der Marknagelung

G. Küntscher

Herausgeber: K. Klemm

Karger, Basel 1986

Reprint der 1. Auflage von 1962 (Schattauer Stuttgart).

Er enthält die handschriftlichen Korrekturen und Anmerkun-

gen, welche der Herausgeber, Prof. Gerhard Küntscher, vor

seinem Tode für eine geplante Neuauflage vorgesehen hatte.

X + 352 S. , 507 Abb. , Leinenband mit Goldprägung,

DM 150

Die von Gerhard Küntscher noch handschriftlich überarbei-tete bisher unveröffentlichte 2. Ausgabe aus dem Jahre 1972, welche unmittelbar vor seinem Tode entstand, wurde jetzt in dankenswerter Weise von Klaus Klemm, Frankfurt/Main, her-ausgegeben. Wer das Werk Gerhard Küntschers kennt, der spurt den besonderen Reiz dieser Faksimile-Ausgabe, die als ein Vermächtnis an die nachfolgenden Generationen angese-hen werden kann, nicht nur, weil der Herausgeber der Ansicht

Atlas der Rectoskopie und Coloskopie

3. neubearbeitete Auflage

Herausgeber: P. Otto, K. Ewe

Springer, Berlin, Heidelberg, New York, Tokyo 1984

XIV + 110 Seiten, 128 vierfarb. Abb. in 22 Tafeln

und 29 Textabb., gebunden DM118,-

ISBN 3-540-12941-3

Der vorliegende «Atlas der Rectoskopie und Coloskopie» von P. Otto und K. Ewe bietet in hervorragender Weise eine Zusammenfassung der Techniken und Ergebnisse dieser wich-tigen endoskopischen Untersuchungsmethode. Es werden übersichtlich die Patientenvorbereitung, das Instrumentarium, die Untersuchungstechniken sowie die Befunddokumentation erläutert. Anhand qualitativ hochwertiger Bilddokumente werden eindrucksvoll und übersichtlich morphologische Befunde dargestellt. Die Literaturnachweise am Ende eines jeden Kapitels ermöglichen Interessierten eine schnelle Wei-terbildung. Somit ist das vorliegende Werk als Einführung für den lernenden Kollegen als auch als Bildduden für den erfah-renen Untersucher sehr empfehlenswert.

H. W. Bauer, Berlin 